\title{
Ergomechanic: A markerless motion capture and ergonomic assessment tool developed using the Workspace workflow engine
}

\author{
R. C. Z. Cohen (D) and S. M. Harrison (D) \\ CSIRO Data61, Clayton, Victoria, Australia \\ Email: Raymond.Cohen@csiro.au
}

\begin{abstract}
Motion capture of human activities is the process of generating a digital representation of the human body performing physical movements. It produces kinematic data, which are typically time series of body joint positions in either world or local body coordinate frames. Motion capture has applications to a range of industry sectors including biomechanics research, rehabilitation, ergonomics, film and sports. Traditional industry standard marker-based motion capture systems (e.g. Vicon Motion Capture System, Vicon, USA) have good spatio-temporal accuracy for detecting reflective markers placed on the body using infra-red cameras. However, this marker-based approach has a number of downsides including the high cost and space requirements of the systems; high time and expertise required to attached markers to each subject; and technical processes of post-processing marker positions to generate the kinematics data. These factors tend to limit both the volume of subjects and trials that can be considered, and the overall uptake of the technology across different sectors. Recently the fields of camera calibration, computer vision, and biomechanics have been combined to produce markerless motion capture (MMC), which can measure human movement without the need for placing any markers (or devices) on the body. When combined with biomechanical simulation, MMC can be used to calculate the number of repetitions of body movements, the speeds and accelerations of movement, and the resulting loading on the internal body structures.
\end{abstract}

Here we describe development of a prototype MMC software that was enabled by the use of computational workflows, specifically the Workspace workflow engine (Cleary et al., 2020). The software prototype, called Ergomechanic, is an MMC and ergonomic assessment tool (Figure 1). Code components from previous projects were immediately available for re-use due to the modular design of operations in Workspace. Interfacing with external libraries such as OpenCV was made robust and simple. Workspace's design facilitated an agile design process, during which the achievable ends were continuously evaluated and refined. Specifically, Workspace gives the designer the ability to: drag and drop or rearrange unit operations, interrogate any input or output of an operation in a suitable widget, use a well-featured OpenGL rendering engine, and a software packaging wizard for deployment. As a result, the current prototype has been developed in a relatively small amount of time and its underlying components can be easily redeployed for other software products with minor additional effort. Along with the software development, this paper highlights a case study of how to use the Ergomechanic platform to record human walking trials, process this data into detailed kinematic information and to objectively assess normal and pathological walking gaits.

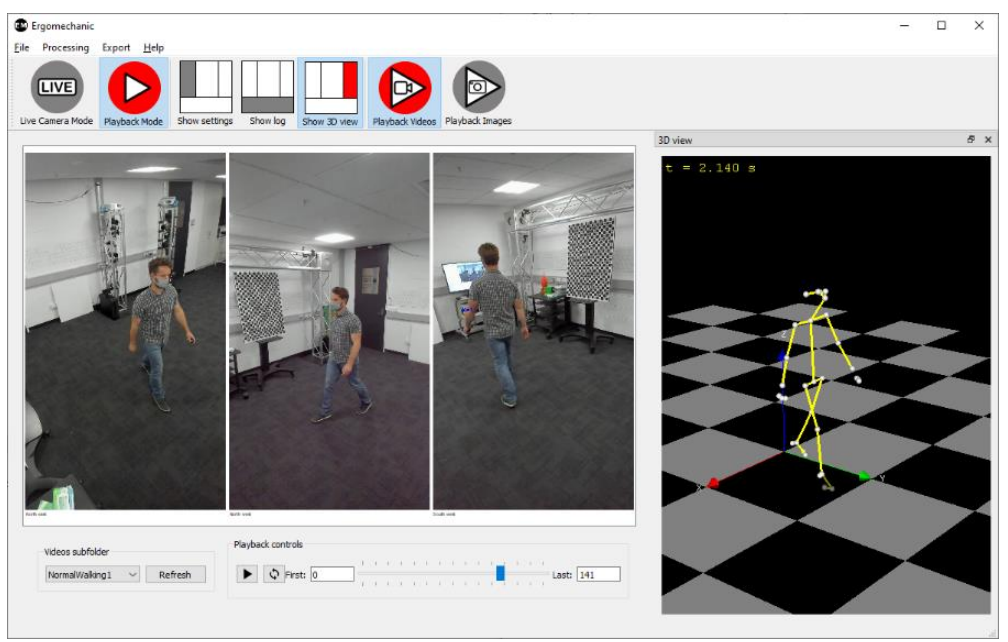

Figure 1. Ergomechanic software interface showing a normal walking trial

Keywords: Workflow, software engineering, markerless motion capture, walking gaits, biomechanics 


\section{INTRODUCTION}

Motion capture of human activities is the process of generating a digital representation of the human body performing movements. It produces kinematic data, which are typically time series of body joint positions in world coordinates (positions and speeds) or in body coordinates (joint angles and angular speeds). Motion capture has applications to a range of industry sectors including biomechanics research, rehabilitation, ergonomics, film and sports. Traditional industry standard marker-based motion capture systems (e.g. Vicon Motion Capture System, Vicon, USA) have very good spatio-temporal accuracy at detecting reflective markers placed on the body using infra-red cameras. However, this marker-based approach has a number downsides including the high cost and space requirements of the systems; high time and expertise required to attached markers to each subject; and technical post-processing of marker data to generate kinematic data. These factors tend to limit the volume of subjects and trials that can be considered and the overall uptake of the technology across different sectors.

Recently the fields of camera calibration, computer vision, and biomechanics have been combined to produce markerless motion capture (MMC), which can measure human movement passively and non-invasively, without the need for placing any markers on the body (Colyer et al., 2018). When combined with biomechanical simulation, MMC can be used to calculate the number of repetitions of body movements, the speeds and accelerations of movement, and the resulting loading on the internal body structures. This new data can be used to inform safer practices and training in the workplace, rehabilitation progression and improvements in sporting technique.

This paper describes the development of a prototype MMC software using the Workspace workflow engine (Cleary et al., 2020). The software, called Ergomechanic, records multi angle video footage of human activities, which it automatically converts to 3D kinematics. The benefits and limitations of using Workspace for development are explored in detail throughout this paper and a case study of its application to human gait assessment is presented. Additional details of the underlying computational algorithms of Ergomechanic are available in Harrison and Cohen (2021) and this paper is complemented by the digital twin modelling papers of Harrison et al. (2021) and Watkins et al. (2021).

\section{METHODS}

This methods section describes the details of the software architecture and development; the trial activity recording; and the trial data postprocessing.

\subsection{Software architecture and development}

Ergomechanic was developed as standalone desktop software utilising GPU hardware and multiple synchronised webcams (many brands and models are supported) to suit many potential industry applications.

\section{Agile design using Workspace}

The use of the CSIRO Workspace workflow engine (Cleary et al., 2020) facilitated an agile design process, during which the achievable ends were not clear but were reviewed and refined through the design and implementation process. The features which helped to enable this were:

- Modularity: Unit operations in Workspace adhere to a well defined structure with inputs and outputs that interface with other workflow operations. The structure of the operation source code also enforces this modular design on plugin developers, regardless of their software design experience. As the requirements of an application workflow changed during agile development, operations provided a suitable level of flexibility. Additionally capability in plugin operations provides long-term benefit by being reusable across different projects, applications and teams.

- Visual programming: The Workspace workflow editor allows the designer to add operations from an Operation Catalogue into the workflow canvas in an intuitive manner; connect and rearrange unit operations; interrogate any operation input or output in a suitable widget; and to visually understand the workflow logic. Therefore updating and refining workflows is easy for both novice and advanced developers.

- Dual purpose: Workspace can be used to develop complex workflows in a research context for a small number of technical users. However the same plugins and workflows can be used to underpin a professional grade application with a suitably simplified graphical user interface (GUI) where the underlying complexity is hidden. This allows pure research and software commercialisation to occur simultaneously. 


\section{Plugins}

The Ergomechanic software required the use of three custom plugins developed for Workspace, each written in $\mathrm{C}++$ and containing their own operations, data structures and widgets. These were:

1. Bio-Mechanic Plugin: This is a general-purpose biomechanical modelling plugin with standard biomechanics data structures and operations for inverse kinematics, forward and inverse dynamics and visualisation. It was originally developed for use in the Dive Mechanic software (Cohen et al., 2020), which was delivered to elite divers in preparation for the Rio Olympics. The plugin's re-use for Ergomechanic demonstrates the continued benefits derived from using the Workspace platform. Further details of this plugin are available in Harrison and Cohen (2019).

2. OpenCV Plugin: This is a computer vision plugin that exposes much of the functionality and data structures of the OpenCV library (2021). Additional Workspace widgets were developed to enable convenient inspection of core data types (e.g. cv::Mat). Key elements used in Ergomechanic were the low-level webcam support, image capture, video I/O capabilities and camera calibration algorithms.

3. Motion Capture Plugin: This plugin provided the critical capability of identifying human pose in twodimensions from optical images. There are a several openly available GPU accelerated Deep Learning models for achieving this including OpenPose (Cao et al., 2019) and Detectron2 (Wu et al., 2019), both of which the plugin supports (see Harrison et al., 2021 for a comparison of pose estimation models). The plugin has been designed to be agnostic to the choice of model, so they can be interchanged depending on the project needs of whole or part body detection; research or commercial use; and internally developed or open-source models.

\section{Workflows}

The main workflow of the Ergomechanic application is shown in Figure 2. The connections between operations define a dependency tree, which Workspace uses to efficiently determine which operations need to be run. This workflow has two main modes of operation. The first mode was "Live Camera Mode" where the workflow would continuously pull in frames from the webcams, which was facilitated by the "While loop". The second mode was a "Playback Mode" where the application would load frames from video files on disk and the "While loop" would be disabled. Variables throughout the workflow enabled control of downstream processing of these video frames. This included 2D pose estimation, 3D reconstruction, video footage overlays and 3D visualisation. Critical variables were tagged as "Global variables" which could be exposed to the user via the GUI.

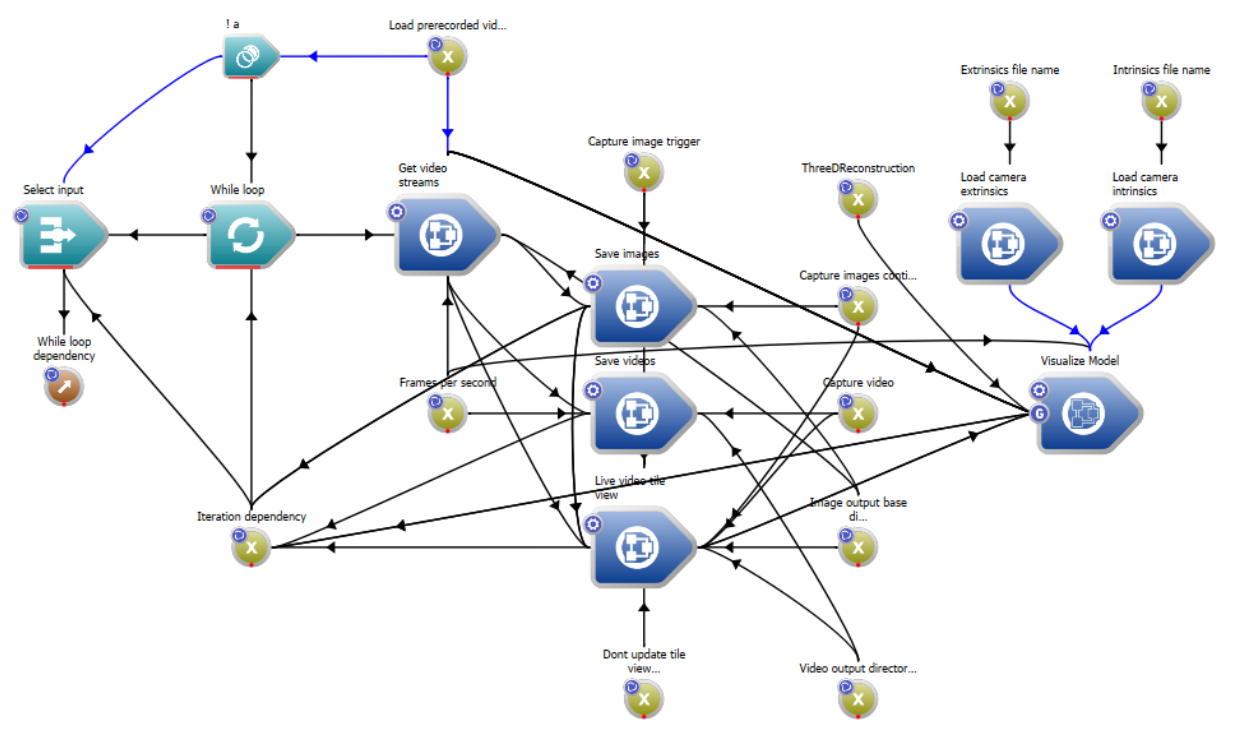

Figure 2. Ergomechanic main workflow. Yellow circles are variables, aqua shapes are operations, blue shapes are nested workflows and the brown circle is the output dependency. Black lines show connections between objects with the data flow direction indicated by the arrows. 


\section{Graphical user interface}

The graphical user interface (GUI) for the software was required to be simple and intuitive for users of all technical abilities. The main features of the GUI are shown in Figure 3 and included:

- File menu: For loading and saving projects.

- Mode selection: For selecting Live Camera Mode or Playback Mode.

- Settings: For providing project video data folder information, camera information, processing flags and visualisation options.

- Trial selection: For selecting a single trial from the current project (Playback Mode only).

- Footage: Live or pre-recorded video streams from the multiple cameras. Overlays of detected features and $3 \mathrm{D}$ reprojections are also available.

- $\quad$ Playback controls: For easy navigation through trial footage.

- 3D view: Gives a rendered view of the 3D reconstruction that can be rotated and zoomed using the mouse.

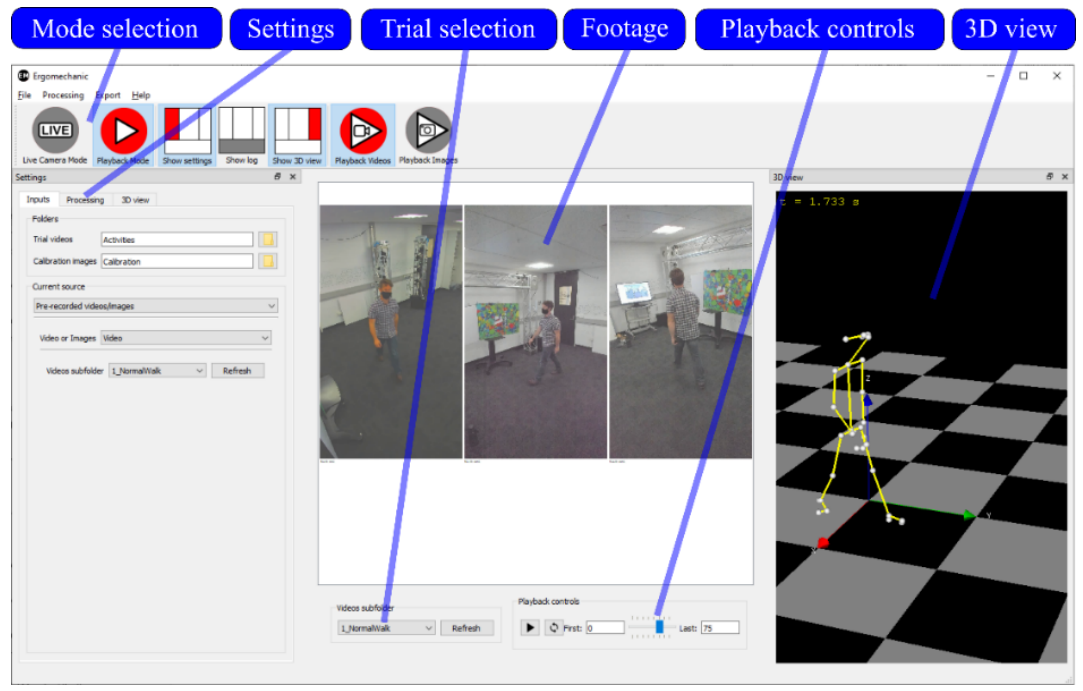

Figure 3. Ergomechanic graphical user interface (GUI) with key elements highlighted.

\section{Application development}

The main elements for the Ergomechanic application development are shown in Figure 4. The application is built upon Workspace and other third party libraries. It requires a number of in-built plugins (e.g. for 3D OpenGL rendering) as well as a number of custom plugins developed by the authors (Bio-Mechanic, Motion capture and OpenCV plugins) and associated third party libraries. A thin application layer sits on top of this comprised of an application workflow, a graphical user interface (GUI) and minimal application C++ code. The application code is mainly responsible for loading the workflow and GUI, connecting global variables to widgets in the GUI, and saving/loading project files. This general structure can be used for any Workspace based application of similar complexity. For this reason Workspace has a wizard for creating a simple application, which guides a developer through the application setup process.

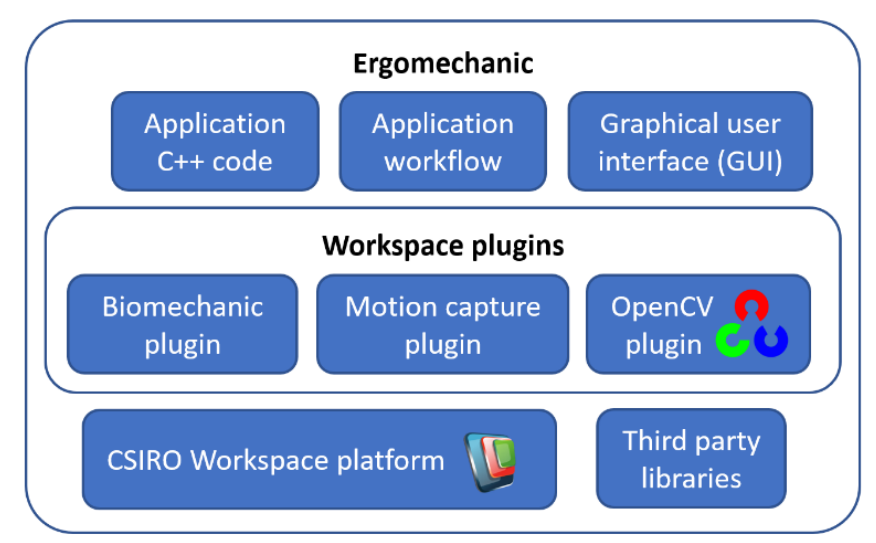

Figure 4. Schematic showing the components of the Ergomechanic application. 


\subsection{Trial activity recording}

\section{Room and equipment setup}

Ergomechanic was installed on a Desktop PC within an indoor laboratory space at CSIRO. Four HD webcams were connected to the PC, positioned around the room and mounted to observe a central "working space" from unique angles within which the trial participant would perform all of their movements.

\section{Camera system calibration process}

It was necessary to calibrate the cameras to determine their focal length and camera distortion (intrinsic parameters) along with their position and orientation (extrinsic parameters). This was done using a ChArUco board consisting checkerboard pattern with inlaid ArUco markers (Muñoz-Salinas and Garrido-Jurado, 2021). The combination of sub-pixel accuracy of checkerboard corner detection algorithms and robust unique identification of the nearby Aruco markers (Figure 5a) makes these boards suitable for camera calibration procedures. The board was placed around the room in 20-30 different positions (visible to most cameras simultaneously) and photos were taken by each camera (Figure $5 \mathrm{~b}$ ). This image set was then passed through OpenCV camera calibration operations to obtain the calibration data for each camera along with the reprojection error, which is indicative of the calibration accuracy.
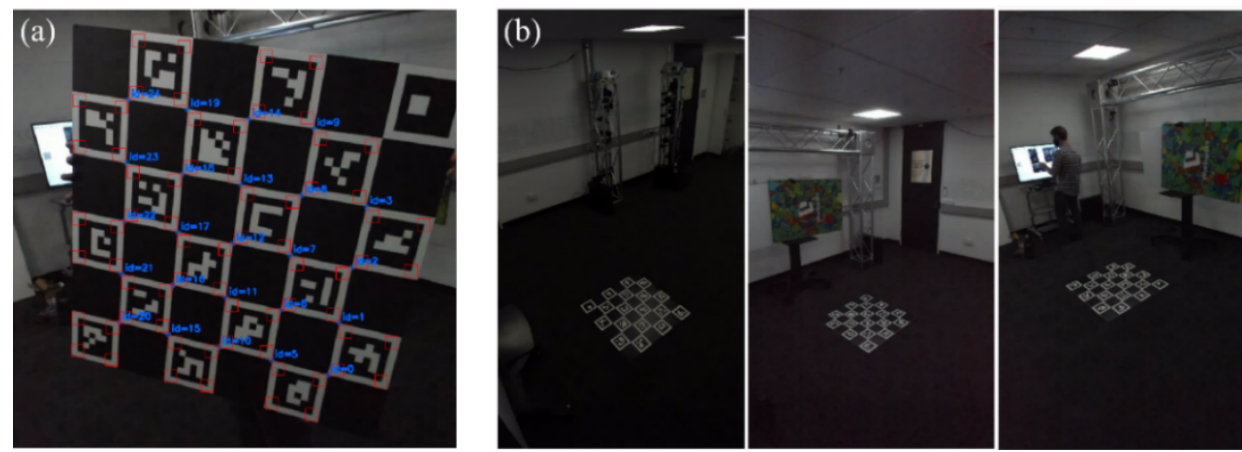

Figure 5. Camera calibration process involving use of Charuco board, (a) Board with automatically detected and identified ArUco corners and (b) multi-camera view of the board.

\section{Trial recording}

One able bodied male trial participant gave informed consent to a procedure approved by the CSIRO ethics board. He completed a number of trials involving walking, shadow boxing, martial arts, and body weight exercises. The cameras were set to record at 30 frames per second at $1280 \times 720$ resolution, which was adequate to capture the spatial and temporal details of the trial activities. The participant started and stopped each trial recording himself using a remote USB "clicker" device, which was connected to the PC. During recording the video streams were buffered into memory and afterwards written to disk as AVI video files with XVID compression. Buffering the videos to memory helped to maximise recording frame rate, which is impacted by video compression and hard disk I/O.

\subsection{Trial data postprocessing}

A human skeletal model was scaled to the anthropometric dimensions of the trial participant by calculating and averaging recorded joint lengths for a subset of the recorded trials and following the methodology of Dumas et al. (2007). The synchronised multi-angle videos recorded during each trial were passed through the $2 \mathrm{D}$ pose estimation model and underwent 3D joint triangulation using the camera calibration data (details available in Harrison et al., 2021). This model was then used in an inverse kinematics algorithm to determine the body joint angles, which would ensure the model joints matched the locations of the $3 \mathrm{D}$ triangulated joints for each frame of the trials. The resultant kinematics consisted of a time series of joint angles for the participant body model.

\section{RESULTS}

Visualisation of the results for the normal walking trial are shown in Figure 6. Each panel shows 2 angles of the video footage with the 3D reconstruction overlaid and rendered alongside. The symmetry of the gait and equal stride lengths are evident in this visualisation. Visualisation of the results from the same participant performing a simulated pathological walking trial are shown in Figure 7. This highlights the asymmetry of the walking gait where the right knee has a limited range of motion (never passing ahead of the left knee) and the entire body is moved differently to compensate for this. Figure 8 shows the time series of the flexion angle of 
the right knee both trials. The range of motion of the right knee for the pathological gait trial is about 25 degrees less than for the normal walking trial.

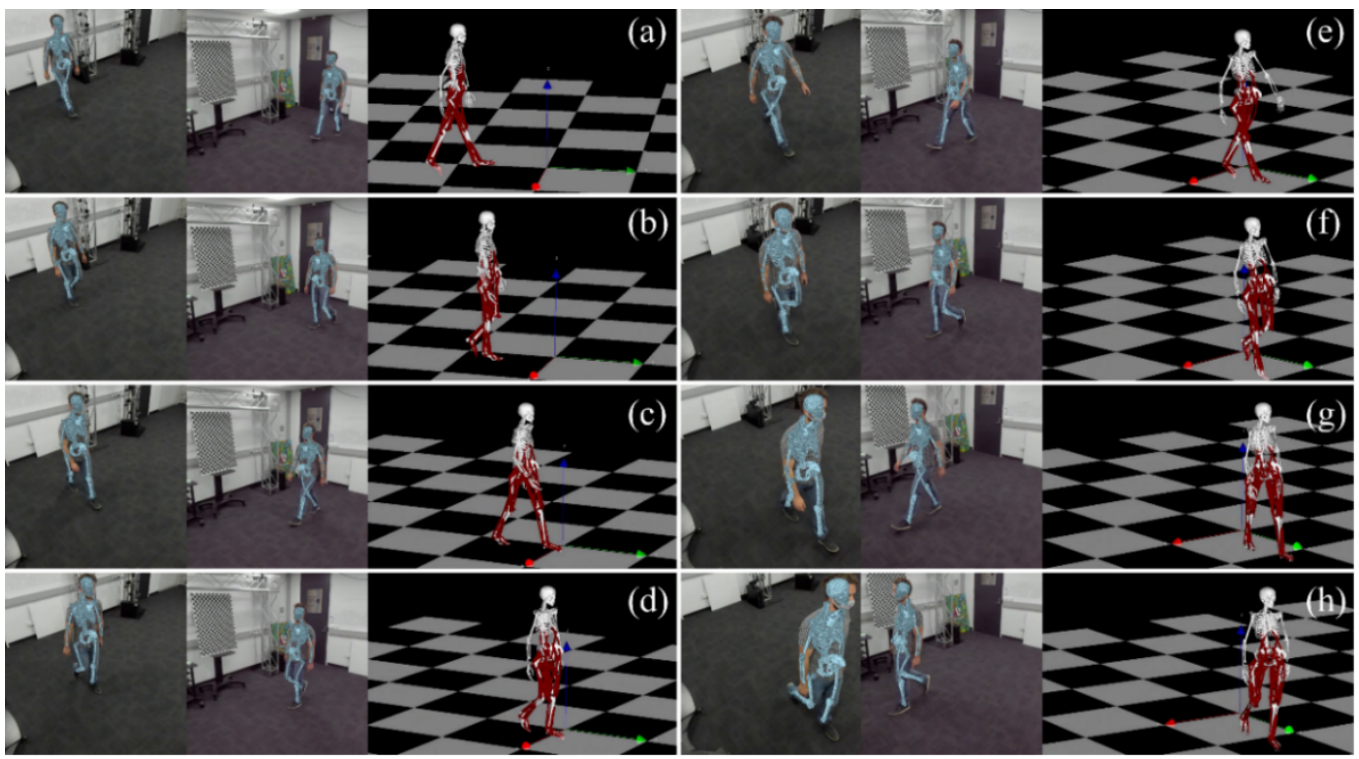

Figure 6. Normal walking trial showing 2 angles of video footage and corresponding 3D motion capture at sequence of key event times of (a) right heel strike, (b) left toe off, (c) left heel strike, (d) right toe off, (e) right heel strike, (f) left toe off, (g) left heel strike, and (h) right toe off.

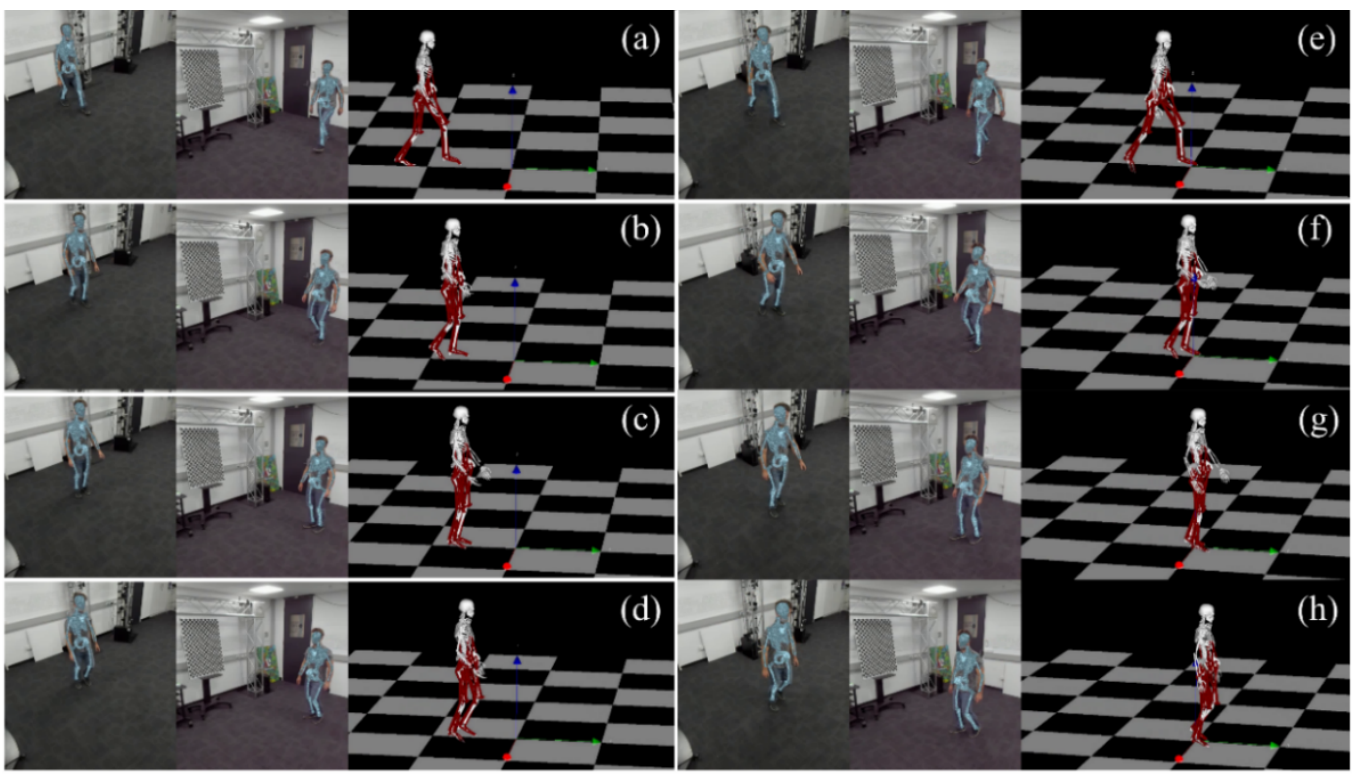

Figure 7. Pathological walking trial showing 2 angles of video footage and corresponding 3D motion capture at sequence of key event times of (a) left heel strike, (b) right toe off, (c) right heel strike, (d) left toe off (e) left heel strike, (f) right toe off, $(\mathrm{g})$ right heel strike, and (h) left toe off.

\section{CONCLUSIONS}

\subsection{Benefits of using workflows for Ergomechanic development}

The use of Workspace for the development of Ergomechanic had several prominent realised benefits:

1. Avoidance of replication: The inherent modularity of plugins, operations and the code base ensured that capability could be easily reused from earlier projects that were developed by small teams of research scientists who typically aren't as adept at software engineering.

2. Efficient development: The creation of a GUI driven application (with a professional appearance) was straightforward to do using the Workspace wizard. The application workflow was intuitive to create 
in the Workspace editor and the choice of which variables to expose through to the GUI could be easily and iteratively refined.

3. Higher performance: Workflows only update operations when strictly required to by the dependency tree, so complex application size workflows (like Ergomechanic) still operate efficiently. Additionally, the use of $\mathrm{C}++$ leads to efficient algorithm development without hidden overheads because of its low level machine control.

\subsection{Insights from the trial results}

The trial results demonstrated that the walking gaits of an individual can be analysed successfully using Ergomechanic without needing markers. The gaits can be inspected visually from any angle using the 3D reconstruction of their skeletons. Additional details of individual joint angles can be quantified and compared. This level of detail of gait information that is useful in studies of human rehabilitation (especially knee and hips) and assessment of individuals with acquired brain injuries (e.g. strokes and other trauma).

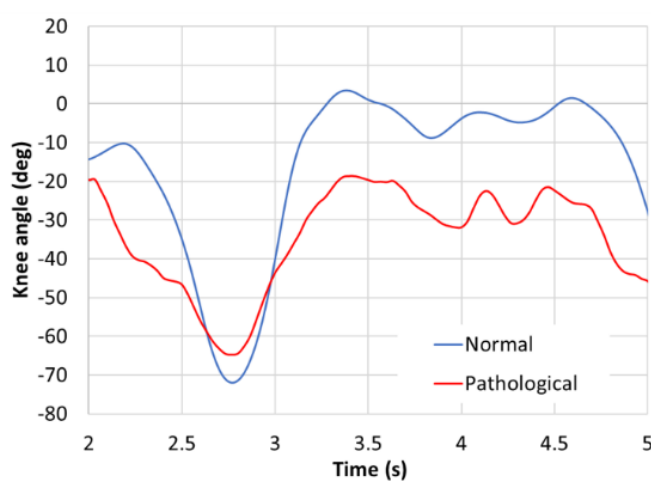

Figure 8. Time series of the right knee angle for both the normal walking trial and the pathological walking trial. Note that the pathological trial has been time-shifted so the minimum right knee angle events coincide.

\section{REFERENCES}

Cao, Z., Hidalgo, G., Simon, T., Wei, S.E., Sheikh, Y., 2019. OpenPose: realtime multi-person 2D pose estimation using Part Affinity Fields. IEEE transactions on pattern analysis and machine intelligence 43, 172-186.

Cleary, P.W., Thomas, D., Hetherton, L., Bolger, M., Hilton, J.E., Watkins, D., 2020. Workspace: a workflow platform for supporting development and deployment of modelling and simulation. Math. Comput. Simulat. 175, 25-61.

Cohen, R.C.Z., Harrison, S.M., Cleary, P.W., 2020. Dive Mechanic: Bringing 3D virtual experimentation using biomechanical modelling to elite level diving with the Workspace workflow engine. Math. Comput. Simulat. 175, 202-217.

Colyer, S.L., Evans, M., Cosker, D.P., Salo, A.I.T., 2018. A review of the evolution of vision-based motion analysis and the integration of advanced computer vision methods towards developing a markerless system. Sports Medicine - Open 4, 1-15.

Dumas, R., Chèze, L., Verriest, J.-P., 2007. Adjustments to McConville et al. and Young et al. body segment inertial parameters. Journal of Biomechanics 40, 543-553.

Harrison, S.M., Cohen, R.C.Z., 2021. Mechanical digital twinning of the human body in the workplace for reduced injury risk and improved health. Presented at the 24th International Congress on Modelling and Simulation (MODSIM2021), Sydney, Australia.

Harrison, S.M., Cohen, R.C.Z., 2019. The Bio-Mechanic Workspace plugin: enabling biomechanical measurement and simulation across workflows and software applications. Presented at the 23rd International Congress on Modelling and Simulation, Canberra, ACT, Australia,.

Harrison, S.M., Cohen, R.C.Z., Cleary, P.W., 2021. Whole body movement modelling in realistic environments for understanding performance and injury, in: Digital Human Modelling and The Digital Twin Patient. Elsevier.

Muñoz-Salinas, R., Garrido-Jurado, S., 2021. ArUco Library [WWW Document]. URL http://sourceforge.net/projects/aruco/

OpenCV Library [WWW Document], 2021. URL https://opencv.org/

Watkins, D., Bolger, M., Hetherington, L., Zhang, Z., Xie, P., Harrison, S.M., Cohen, R.C.Z., 2021. Using Workspace to Implement Digital Twins in the Mixed Reality Lab. Presented at the 24th International Congress on Modelling and Simulation (MODSIM2021), Sydney, Australia.

Wu, Y., Kirillov, A., Massa, F., Lo, W.Y., Girshick, R., 2019. Detectron2 [WWW Document]. URL https://github.com/facebookresearch/detectron2 